\title{
Observation of Squeezed Light with 10-dB Quantum-Noise Reduction
}

\author{
Henning Vahlbruch, Moritz Mehmet, Simon Chelkowski, Boris Hage, Alexander Franzen, Nico Lastzka, Stefan Goßler, \\ Karsten Danzmann, and Roman Schnabel \\ Institut für Gravitationsphysik, Leibniz Universität Hannover and Max-Planck-Institut für Gravitationsphysik \\ (Albert-Einstein-Institut), Callinstr. 38, 30167 Hannover, Germany
}

(Received 13 August 2007; published 23 January 2008)

\begin{abstract}
Squeezing of light's quantum noise requires temporal rearranging of photons. This again corresponds to creation of quantum correlations between individual photons. Squeezed light is a nonclassical manifestation of light with great potential in high-precision quantum measurements, for example, in the detection of gravitational waves [C. M. Caves, Phys. Rev. D 23, 1693 (1981)]. Equally promising applications have been proposed in quantum communication [H. P. Yuen and J. H. Shapiro, IEEE Trans. Inf. Theory 24, 657 (1978)]. However, after 20 years of intensive research doubts arose whether strong squeezing can ever be realized as required for eminent applications. Here we show experimentally that strong squeezing of light's quantum noise is possible. We reached a benchmark squeezing factor of 10 in power $(10 \mathrm{~dB})$. Thorough analysis reveals that even higher squeezing factors will be feasible in our setup.
\end{abstract}

Theoretical considerations about the possible existence of light with squeezed quantum noise can be traced back to the 1920's [1]. However, only after applications for squeezed light were proposed in the 1980's squeezing was discussed in more detail [1-5]. In [2] it was suggested to use squeezed light to improve the sensitivity of kilometre-scale Michelson laser-interferometers for the detection of gravitational waves. Such detectors have now reached a technical standard at which squeezed light can contribute in a valuable way. For example squeezing the quantum noise can provide a sensitivity improvement equivalent to even higher laser powers, however, without increasing the already problematic thermal load inside the interferometer. This is of great relevance for cryogenically cooled detectors. Proof of principle experiments have been successfully conducted [6,7] and squeezed states have been generated also in the audio signal band of ground-based detectors [8,9]. Another field of application is continuousvariable $(\mathrm{CV})$ quantum communication and information $[3,10,11]$. While discrete-variable quantum information typically relies on single-photon detectors, which are limited in terms of detection speed and quantum efficiency, squeezed light is detected with homodyne and heterodyne detectors which reveal quantum correlations by averaging over a vast number of detected photons. Because of this, high bandwidth and almost perfect detection efficiencies are possible. Squeezed states of light have been used to demonstrate several CV quantum information protocols. They have been used to construct entangled states of light and to demonstrate quantum teleportation [12-14]. They are a possible resource for secure quantum key distribution protocols $[15,16]$ and for generation of cluster states for universal quantum computing [17]. Recently, squeezed states of light have been used to prepare macroscopic quantum superposition states for quantum information networks $[18,19]$.
For all proof of principle experiments so far only modest strengths of squeezing were available. In [6-9] about 3 to $4 \mathrm{~dB}$ of squeezing was achieved. The first $\mathrm{CV}$ teleportation experiments $[12,13]$ did not reach the so-called no-cloning limit of fidelity greater than $2 / 3$ [20] due to the limitations in squeezing strength. Observation of much stronger squeezing indicates that high purity squeezed states can be produced, for example, to beat the Holevo capacity limit of quantum communication channels [21]. Quite generally, the applications of squeezed light become more momentous with stronger squeezing. Although the first experimental demonstration of squeezed light succeeded in 1985 [22], dedicated research in the following two decades could only elaborate typical factors of 2 to 4 (3 to $6 \mathrm{~dB}$ ), see also [23,24]. However, very recently a great step forward was achieved at the University of Tokyo and a factor of $8(9 \mathrm{~dB})$ quantum-noise squeezing of a laser field at $860 \mathrm{~nm}$ was observed [25]. This wavelength is close to atomic transitions having important implications for quantum information storage [26]. In our experiment we generated a squeezed laser beam with a quantum noise reduction of a factor of 10 at a laser wavelength of $1064 \mathrm{~nm}$ which is used in current gravitational wave detectors [27].

As shown in Fig. 1 the laser source of our experiment was a monolithic nonplanar Nd:YAG ring laser of $2 \mathrm{~W}$ continuous wave single-mode output power at $1064 \mathrm{~nm}$, the wavelength used for the current generation of gravitational wave detectors. Approximately $1.9 \mathrm{~W}$ were used for second harmonic generation (SHG) to provide the pump field at $532 \mathrm{~nm}$ for our optical parametric squeezed light source. A detailed description of the SHG design can be found in [28]. An important feature of our experiment were two traveling-wave resonators which served as optical lowpass filters for phase noise on the laser beams as well as spatial mode cleaners. These cavities were positioned in 


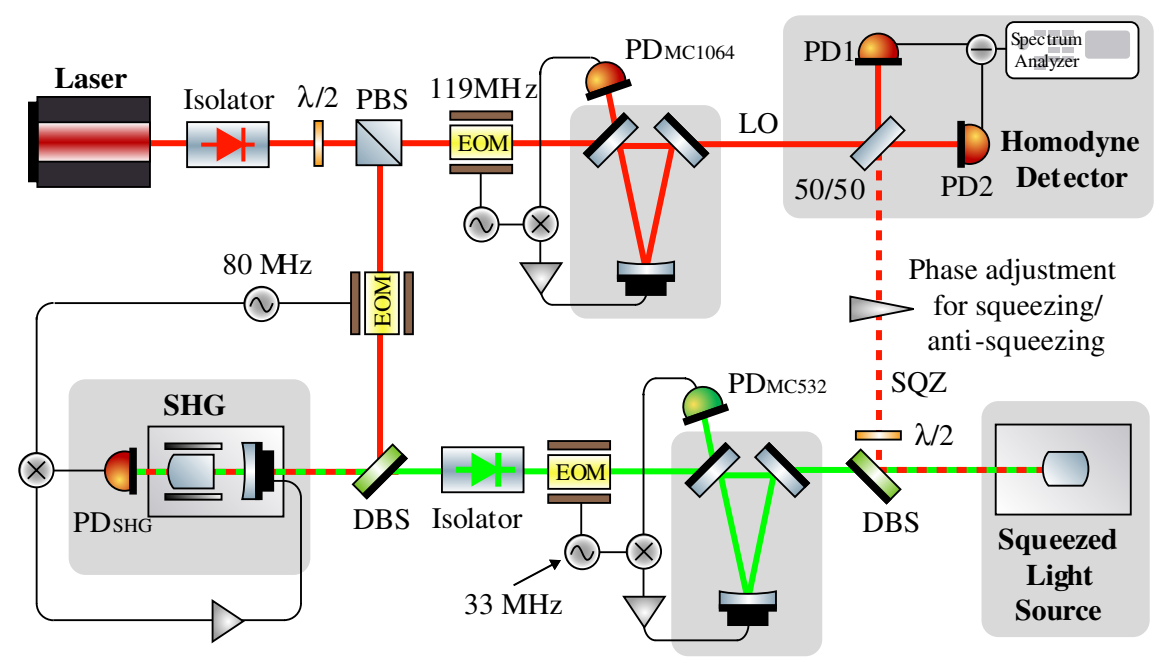

FIG. 1 (color online). Schematic of the experimental setup. Squeezed states of light (SQZ) at 1064 nm were generated by type I optical parametric oscillation (OPO) below threshold. SHG, Second harmonic generation (SHG); Polarizing beam splitter (PBS); Dichroic beam splitter (DBS); Local oscillator (LO), photodiode (PD); electro-optical modulator (EOM).

the beam path of both the fundamental and second harmonic field; one cavity close to the homodyne detector and one close to the squeezed light source. Both resonators had a finesse of 350 and a linewidth of $1.44 \mathrm{MHz}$. The cavities were held on resonance with the laser fields via a PoundDrever-Hall locking scheme with phase modulation frequencies of 119 and $33 \mathrm{MHz}$. These resonators significantly reduced phase front mismatches and phase fluctuations. Both, spatial and temporal fluctuations lead to a reduced contrast between the squeezed field and the local oscillator beam of the homodyne detector. This effect couples antisqueezed noise into the squeezing measurement. It has been shown in $[25,29]$ that phase fluctuations, for example, of the second-harmonic pump field, can be a limiting factor for strong squeezing. For both resonators we optimized the phase modulation depth for an optimal balance between the signal-to-noise ratio of the error signal and the residual phase modulation of the transmitted light.

Our squeezed light source was a monolithic cavity made from $7 \%$ doped $\mathrm{MgO}: \mathrm{LiNbO}_{3}$ that produced squeezed states via type I degenerate optical parametric oscillation (OPO). The crystal length was $6.5 \mathrm{~mm}$ and both front and rear face had a radius of curvature of $8 \mathrm{~mm}$. Each surface was dielectrically coated to give power reflectivities of $88 \%$ or $99,97 \%$ at $1064 \mathrm{~nm}$, respectively. Thus the waist size radius of the infrared field was approximately $28 \mu \mathrm{m}$. Taking the refractive index of $n=2.23$ into account, this resulted in a cavity free spectral range of approximately $10.34 \mathrm{GHz}$ and a cavity-linewidth of $210 \mathrm{MHz}$. The mirror coatings on the crystal also provided a second harmonic power built up by a factor of approximately 3 when the fundamental wavelength was resonant. Second harmonic pump powers between 650 and $950 \mathrm{~mW}$ were modematched into the squeezed light source and parametric gains between 63 to more than 200 were observed.
Squeezed states were produced when the crystal temperature was stabilized at its phase-matching temperature and the laser wavelength was tuned on resonance with the squeezed light source cavity. Because of the high stability of our setup no servo-loop control for the laser frequency was required. The squeezed states left the source in the counter direction of the pump field and were separated via a dichroic beam splitter (DBS). The observation of (squeezed) quantum noise was performed by means of a balanced homodyne detector built from a pair of Epitaxx ETX-500 photodiodes with a detection bandwidth of approximately $50 \mathrm{MHz}$. We achieved a fringe visibility of $99.8 \%$ between the squeezed beam and the local oscillator on the 50/50 homodyne beam splitter.

Figure 2 presents the first ever direct observation of light with $10 \mathrm{~dB}$ squeezing. Shown are noise powers at the Fourier sideband frequency of $5 \mathrm{MHz}$. Trace (a) corresponds to the shot-noise of uncorrelated photons of $26.9 \mathrm{~mW}$ local oscillator power and was measured with the squeezed light input blocked. In this arrangement no photons entered the signal port of the homodyne detector and the measured shot-noise can be directly linked to the vacuum noise, which corresponds to the light's quantum mechanical ground state. Trace (b) shows the quantum-noise reduction when squeezed states were injected. The directly observed squeezing level was 10.12 $( \pm 0.15) \mathrm{dB}$. During this measurement the phase between the squeezed vacuum field and the local oscillator beam of the homodyne detector was adjusted manually via a highvoltage driven piezomounted mirror. This enabled us to measure maximum squeezing levels as well as the maximum antisqueezing. The detector dark noise (trace (c)) was approximately $26 \mathrm{~dB}$ below the vacuum noise level. Dark noise subtraction leads to a squeezing level of 10.22 $( \pm 0.16) \mathrm{dB}$. 


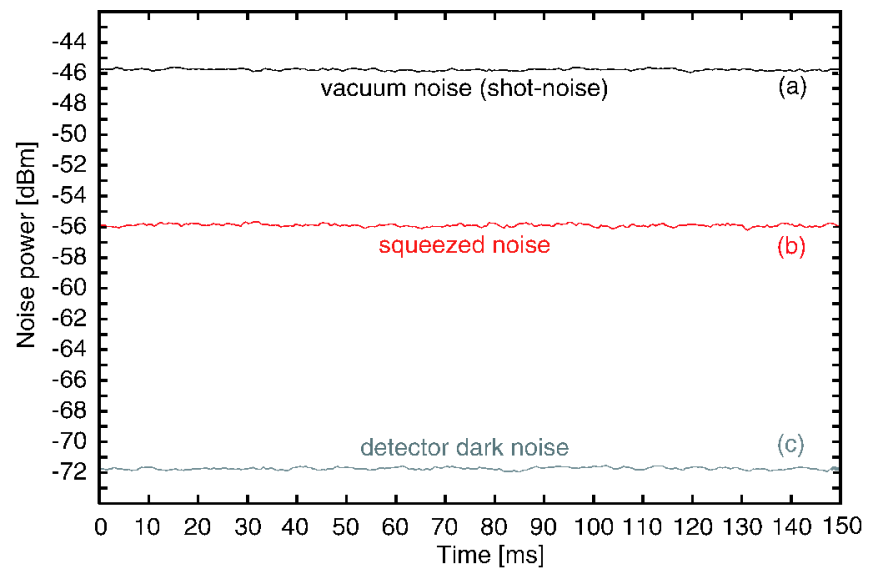

FIG. 2 (color online). Quantum noise powers at a Fourier frequency of $5 \mathrm{MHz}$, measured with a resolution bandwidth of $100 \mathrm{kHz}$ and video bandwidth of $100 \mathrm{~Hz}$. Trace (a) shows the vacuum noise level corresponding to $26.9 \mathrm{~mW}$ local oscillator power. Trace (b) shows the noise power of the squeezed vacuum states measured with the same local oscillator power. A nonclassical noise reduction of $10.12 \mathrm{~dB}$ below vacuum noise was observed. The electronic detector dark noise is shown in trace (c) and was not subtracted from the data. Each trace was averaged 3 times.

To confirm the observed squeezing strength, we checked linearity of the homodyne detection system including the spectrum analyzer by measuring shot-noise levels versus local oscillator powers (Fig. 3). A linear fit matches the measurements accurately. To further validate the observation of $10 \mathrm{~dB}$ squeezing we introduced a known amount of optical loss into the squeezed light beam. The observed

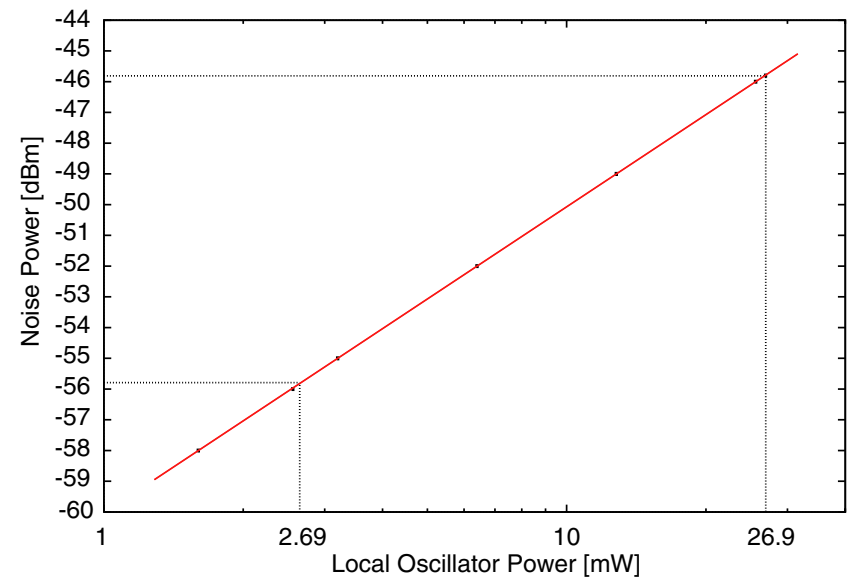

FIG. 3 (color online). The linearity of the homodyne detection system was validated by varying the local oscillator power. Shown is the linear fit to seven measurement values (squares). The sizes of the squares corresponds to the measurement error bars. Note that the shot-noise of a laser beam of $2.69 \mathrm{~mW}$ is shown to be identical to the squeezed noise of the differential mode in our homodyne detector with 10 times the light power, compare with Fig. 1. squeezing and antisqueezing strength should depend on this additional loss in a characteristic way. For this procedure a combination of a $\lambda / 2$ wave plate and a polarizing beam splitter was placed between the $50 / 50$ beam splitter of the homodyne detector and each photodiode (PD1, PD2). Since both fields - the squeezed beam and the local oscillator - suffered from the loss, the intensity of the local oscillator beam was recalibrated to the nominal value of $26.9 \mathrm{~mW}$ by using a more intense beam in front of the mode cleaner. Figure 4 shows the observed amount of squeezing and antisqueezing with an additional $10 \%$, $20 \%, 30 \%$, and $40 \%$ introduced optical loss, respectively. The solid lines (b) and (c) represent the simulations for a parametric gain of $g=63$ which was experimentally realized with $650 \mathrm{~mW}$ pump power. We found excellent agreement with the experimental data.

With an increased pump power of $950 \mathrm{~mW}$ we observed antisqueezing of $23.3 \mathrm{~dB}$ whereas the squeezing was still $10 \mathrm{~dB}$ below vacuum noise. This observation can be used to deduce boundaries for the total optical loss in our setup. Assuming a loss free setup in which the observed squeezing strength is limited by antisqueezing coupling into our squeezing measurement via phase fluctuations, we derived the upper limit for phase jitter to be $\phi=1.2^{\circ}$. Since $\phi$ is independent of the pump power we can conclude that $10 \mathrm{~dB}$ squeezing, as observed with $650 \mathrm{~mW}$ (and less antisqueezing), was not limited by phase fluctuations but optical loss. Even with $\phi=1.2^{\circ}$ we find the minimum value for the total optical loss in our setup to $5.6 \%$. Second, we assumed phase fluctuations of $\phi \ll 1.2^{\circ}$. Here the observed squeezing is completely limited by optical loss, which results in the upper bound of $8.6 \%$. Taking these

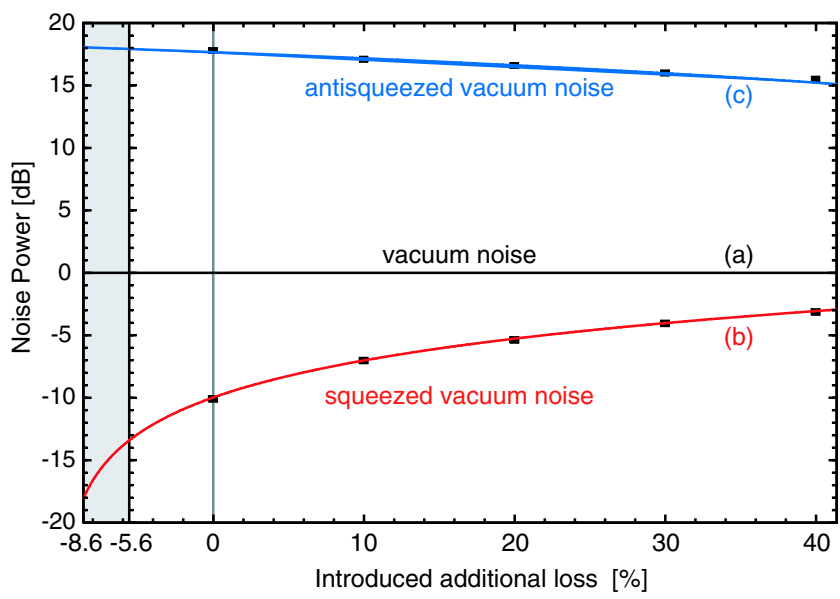

FIG. 4 (color online). Squeezing and antisqueezing levels for a parametric gain of 63 versus optical loss. Solid lines show the theoretical predictions. Square boxes represent measurement values with sizes corresponding to the errors bars. Electronic dark noise was subtracted in this figure. The two vertical axis on the left corresponds to the upper and lower boundaries of how much squeezing might be achieved in our setup by reduction of optical loss. 
boundaries into account, the left part of Fig. 4 shows how much squeezing might be achieved in our setup by optical loss reduction.

In independent measurements we determined the intracavity round trip loss of the squeezed light source at $1064 \mathrm{~nm}$ to be less than $0.07 \%$, corresponding to an escape efficiency of the squeezed states from the source in excess of $99.4 \%$. Loss during propagation occurs due to the dichroic beam splitter $(0,2 \%$ transmission loss at $1064 \mathrm{~nm})$ and nonperfect antireflection coatings of lenses and were determined to be about $1.1 \%$. The nonperfect visibility at the homodyne beam splitter introduced another $0.4 \%$ of loss. Given these values we estimate the quantum efficiency of the ETX-500 photodiodes to be $95( \pm 2) \%$. Our analysis suggests that the nonperfect quantum efficiency of our photodiodes was the main limitation in our experiment. With improved photodiodes close to unity quantum efficiency, which already exist for shorter wavelengths [25], an additional factor of 2 in quantum noise reduction might be possible.

The direct observation of $10 \mathrm{~dB}$ squeezing of quantum noise of light, as reported here, shows that the squeezed light technique has indeed a great application potential as envisaged more than two decades ago. Injected into a gravitational wave detector, the quantum noise reduction corresponding to an increase of factor 10 in laser light power will be possible [2]. This is a promising application, since gravitational wave detectors already use the highest single-mode laser powers applicable. Furthermore, our results might enable the generation of strongly entangled states to reach teleportation fidelities well above $2 / 3$ as already typically achieved in single-photon teleportation experiments [30].

We would like to thank H.-A. Bachor, W. P. Bowen, J. DiGuglielmo, J. Harms, S. Hild, N. Mavalvala, D. E. McClelland, K. McKenzie, P. K. Lam, and A. Thüring for helpful discussions regarding the optimization of squeezed light generation and detection. This work has been supported by the Deutsche Forschungsgemeinschaft and is part of Sonderforschungsbereich 407.

[1] V. V. Dodonov, J. Opt. B 4, R1 (2002).

[2] C. M. Caves, Phys. Rev. D 23, 1693 (1981).

[3] H. P. Yuen and J. H. Shapiro, IEEE Trans. Inf. Theory 24, 657 (1978)

[4] J. N. Hollenhorst, Phys. Rev. D 19, 1669 (1979).

[5] D. F. Walls, Nature (London) 306, 141 (1983).

[6] K. McKenzie, D. A. Shaddock, D. E. McClelland, B. C. Buchler, and P. K. Lam, Phys. Rev. Lett. 88, 231102 (2002).
[7] H. Vahlbruch, S. Chelkowski, B. Hage, A. Franzen, K. Danzmann, and R. Schnabel, Phys. Rev. Lett. 95, 211102 (2005).

[8] K. McKenzie, N. Grosse, W. P. Bowen, S. E. Whitcomb, M. B. Gray, D. E. McClelland, and P. K. Lam, Phys. Rev. Lett. 93, 161105 (2004).

[9] H. Vahlbruch, S. Chelkowski, B. Hage, A. Franzen, K. Danzmann, and R. Schnabel, Phys. Rev. Lett. 97, 011101 (2006).

[10] S. L. Braunstein and P. van Loock, Rev. Mod. Phys. 77, 513 (2005).

[11] D. Akamatsu, K. Akiba, and M. Kozuma, Phys. Rev. Lett. 92, 203602 (2004).

[12] A. Furusawa, J. L. Sørensen, S. L. Braunstein, C. A. Fuchs, H. J. Kimble, and E. S. Polzik, Science 282, 706 (1998).

[13] W. P. Bowen, N. Treps, B. C. Buchler, R. Schnabel, T. C. Ralph, H.-A. Bachor, T. Symul, and P. K. Lam, Phys. Rev. A 67, 032302 (2003).

[14] N. Takei, H. Yonezawa, T. Aoki, and A. Furusawa, Phys. Rev. Lett. 94, 220502 (2005).

[15] R. García-Patrón and N. J. Cerf, Phys. Rev. Lett. 97, 190503 (2006).

[16] M. Navascués, F. Grosshans, and A. Acín, Phys. Rev. Lett. 97, 190502 (2006).

[17] N.C. Menicucci, P. van Loock, M. Gu, C. Weedbrook, T. C. Ralph, and M.A. Nielsen, Phys. Rev. Lett. 97, 110501 (2006).

[18] A. Ourjoumtsev, R. Tualle-Brouri, J. Laurat, and P. Grangier, Science 312, 83 (2006).

[19] J. S. Neergaard-Nielsen, B. Melholt Nielsen, C. Hettich, K. Mølmer, and E. S. Polzik, Phys. Rev. Lett. 97, 083604 (2006).

[20] F. Grosshans and P. Grangier, Phys. Rev. A 64, 010301 (2001).

[21] S.L. Braunstein and H. J. Kimble, Phys. Rev. A 61, 042302 (2000).

[22] R. E. Slusher, L. W. Hollberg, B. Yurke, J. C. Mertz, and J. F. Valley, Phys. Rev. Lett. 55, 2409 (1985).

[23] N. Treps, N. Grosse, W. P. Bowen, C. Fabre, H.-A. Bachor, and P. K. Lam, Science 301, 940 (2003).

[24] G. Breitenbach, S. Schiller, and J. Mlynek, Nature (London) 387, 471 (1997).

[25] Y. Takeno, M. Yukawa, H. Yonezawa, and A. Furusawa, Opt. Express 15, 4321 (2007).

[26] M. T. L. Hsu, G. Hétet, O. Glöckl, J. J. Longdell, B. C. Buchler, H.-A. Bachor, and P. K. Lam, Phys. Rev. Lett. 97, 183601 (2006).

[27] P. Aufmuth and K. Danzmann, New J. Phys. 7, 202 (2005).

[28] S. Chelkowski, H. Vahlbruch, K. Danzmann, and R. Schnabel, Phys. Rev. A 75, 043814 (2007).

[29] A. Franzen, B. Hage, J. DiGuglielmo, J. Fiurášek, and R. Schnabel, Phys. Rev. Lett. 97, 150505 (2006).

[30] D. Bouwmeester et al., Nature (London) 390, 575 (1997); D. Boschi et al., Phys. Rev. Lett. 80, 1121 (1998); I. Marcikic et al., Nature (London) 421, 509 (2003); R. Ursin et al., Nature (London) 430, 849 (2004). 